\title{
Correction to: Characteristics of Japanese patients with Leber's hereditary optic neuropathy and idebenone trial: a prospective, interventional, non-comparative study
}

\author{
Hiroto Ishikawa ${ }^{1}$ (D) Yoichiro Masuda ${ }^{2} \cdot$ Hitoshi Ishikawa ${ }^{3} \cdot$ Keigo Shikishima $^{2} \cdot$ Toshiaki Goseki $^{3} \cdot$ Takeshi Kezuka $^{4}$. \\ Masahiko Terao $^{5} \cdot$ Atsushi Miyazaki $^{6} \cdot$ Kenji Matsumoto $^{6} \cdot$ Hiroki Nishikawa $^{7} \cdot$ Fumi Gomi $^{1} \cdot$ Osamu Mimura $^{1}$
}

Published online: 6 January 2021

(c) Japanese Ophthalmological Society 2020

\section{Correction to: Japanese Journal of Ophthalmology https://doi.org/10.1007/s10384-020-00789-2}

In the original publication of this paper, the name of fourth author should be Keigo Shikishima. The correct version of author name is given in this correction.

Publisher's Note Springer Nature remains neutral with regard to jurisdictional claims in published maps and institutional affiliations.

The original article can be found online at https://doi.org/10.1007/ s10384-020-00789-2.

\section{Hiroto Ishikawa}

ohmyeye@gmail.com

1 Department of Ophthalmology, Hyogo College of Medicine, 1-1 Mukogawa-cho, Nishinomiya, Hyogo 663-8501, Japan

2 Department of Ophthalmology, The Jikei University School of Medicine, Nishi-Shinbashi, Minato-ku, Tokyo, Japan

3 Department of Ophthalmology, Kitasato University School of Medicine, Sagamihara, Kanagawa, Japan

4 Department of Ophthalmology, Tokyo Medical University, Nishi-Shinjuku, Shinjuku-ku, Tokyo, Japan

5 Research Institute for Time Studies, Yamaguchi University, Yamaguchi, Japan

6 Brain Science Institute, Tamagawa University, Machida, Japan

7 Center for Clinical Research and Education, Hyogo College of Medicine, Nishinomiya, Hyogo, Japan 